\title{
Untersuchungsdimensionen und methodisches Vorgehen
}

\subsection{Untersuchungsdimensionen}

Um das entwicklungsbezogene Engagement der KAAD-Studierenden bzw. Alumni vor diesem Hintergrund möglichst differenziert und umfassend zu untersuchen, wurde ein mehrdimensionales und multiperspektivisches Forschungsdesign entwickelt, das es ermöglichen sollte, die verschiedenen Faktoren und Dimensionen, die in diesem Prozess eine Rolle spielen, in den Blick zu nehmen und auch systematisch miteinander zu vergleichen. So macht es für das entwicklungsbezogene Engagement der Stipendiat*innen möglicherweise einen Unterschied, ob sie tatsächlich ins Herkunftsland zurückgekehrt sind oder nicht bzw. wie sie darüber denken. Um ihre Aktivitäten systematisch voneinander unterscheiden zu können, wurden in einem ersten Schritt grundsätzlich zwei Untersuchungsgruppen voneinander unterschieden: KAAD-Alumni, die in ihr Herkunftsland zurückgekehrt sind, und KAAD-Alumni, die nicht in ihr Herkunftsland zurückgegangen sind. Zudem wurden internationale Studierende und Forschende, die aktuell vom KAAD gefördert werden und sich in Deutschland aufhalten, in die Untersuchung aufgenommen, um sie zu ihren aktuellen Aktivitäten sowie zu ihren Erwartungen und Einschätzungen bzgl. einer möglichen Rückkehr zu befragen. Um einen Vergleich zu der Welt außerhalb des KAAD zu ermöglichen, wurde zudem auch eine Gruppe internationaler Studierender in die Untersuchung einbezogen, die nicht vom KAAD gefördert werden, um die Bedeutung der Unterstützungsnetzwerke des KAAD und seiner Partnerorganisationen in Deutschland und in den Herkunftsländern besser einschätzen zu können.

Zudem wurde nach verschiedenen Fachrichtungen differenziert. So gingen wir davon aus, dass es einen Unterschied machen kann, welches Fach man studiert bzw. studierte und welche strukturellen Rahmenbedingungen in den jeweiligen Berufsfeldern im Herkunfts- bzw. im Studienland vorzufinden sind, an die ggf. an-

S. Krannich und U. Hunger, Studierendenmigration und Entwicklung, https://doi.org/10.1007/978-3-658-32048-5_2 
geknüpft werden kann. Im Hinblick auf die verschiedenen Fachdisziplinen wurden drei verschiedene Fächergruppen untersucht:

- MINT-Fächer (Mathematik, Informatik, Naturwissenschaft und Technik). Absolvent*innen aus diesen Fächern spielen gerade aus entwicklungspolitischer Perspektive eine herausgehobene Rolle, etwa wenn es um die Entwicklung und Übertragung neuer Produktionsverfahren und Technologien geht. Diese Gruppe ist zudem auch in den Industrieländern sehr umworben, weshalb ihre Rückkehr ins Herkunftsland noch einmal mehr in Frage gestellt wird, zumal auch sprachliche Barrieren hier weniger bestehen, da vielfach Englisch als Arbeitssprache gilt.

- Medizin und Gesundheitswissenschaften. Auch diese Gruppe stellt eine sehr entwicklungsrelevante Fachgruppe dar. Auf der einen Seite leiden viele Länder bereits heute unter einer enormen gesundheitlichen Unterversorgung und sind ausländische Ärzt ${ }^{*}$ innen in Industrieländern sehr begehrt, so dass ein weiterer Brain Drain in diesem Bereich nicht unwahrscheinlich erscheint und äußerst kritische Auswirkungen haben würde. Auf der anderen Seite könnten Mediziner*innen (und andere im Gesundheitswesen Tätige) durch ein Auslandsstudium oder eine Weiterbildung im Ausland möglicherweise wichtige Fachkenntnisse erwerben, die sie anschließend gewinnbringend in den Entwicklungsprozess ihres Heimatlandes einbringen könnten.

- Rechts-, Wirtschafts-, Geistes-, Kultur- und Sozialwissenschaften. Auch diese Gruppe stellt ein spezifisches Potenzial für die Entwicklung in den Herkunftsländern der Migrant*innen dar, und zwar insbesondere im Hinblick auf die politische, rechtliche und gesellschaftliche Entwicklung insgesamt. So ist der Transfer von demokratischen Werten und Verfahrensweisen (social remittances) mindestens ebenso wichtig wie die Förderung der technologischen Entwicklung und medizinischen Versorgung in den Herkunftsländern.

Schließlich wurde das Untersuchungsdesign auch in religiöser bzw. kirchlicher Hinsicht differenziert, da in den Herkunftsländern auch in religiöser bzw. kirchlicher Hinsicht unterschiedliche Anknüpfungspunkte bzw. Hindernisse für ein entwicklungsbezogenes und religiöses Engagement bestehen. Ein besonderes $\mathrm{Au}-$ genmerk wurde dabei auf die KAAD-Gremien und -Organisationen und andere kirchliche Organisationen gelegt, welche Rolle sie spielen und wie sie das kirchlichentwicklungsbezogene Engagement der Stipendiat*innen unterstützen und prägen. 


\subsection{Qualitative Länderfallstudien}

Alle drei Faktorenbündel bzw. Dimensionen (Rückkehr, Fachrichtungen, Herkunftslandkontexte) wurden anhand von fünf Fallstudienländern, in denen die dargestellten Untersuchungsgruppen und Fragedimensionen mit jeweils unterschiedlichen Schwerpunkten abgebildet wurden, analysiert. Hierbei handelte es sich um Palästina/Israel, Indonesien, Ghana, Georgien und Kolumbien. Im Hinblick auf die unterschiedlichen Fachrichtungen stand Israel/Palästina dabei für den Fall, dass viele Mediziner ${ }^{\star}$ innen zu den Geförderten zählen, Indonesien für einen Schwerpunkt im Bereich MINT, Georgien und Kolumbien für einen Schwerpunkt bei Rechts-, Kultur- und Sozialwissenschaften und Ghana für den Bereich Umwelt und Landwirtschaft.

In den ausgewählten Fallstudienländern waren zudem die religiösen Kontexte unterschiedlich. So ist Kolumbien stark katholisch geprägt, Georgien vor allem georgisch-orthodox und Indonesien muslimisch. In Israel/Palästina leben überwiegend Menschen jüdischen oder muslimischen Glaubens und in Ghana bekennen sich gleich große Teile der Bevölkerung zu christlichen Kirchen, zum Islam und zu traditionellen Religionen. Generell gibt es in allen fünf Ländern (in unterschiedlicher Ausprägung) staatliche und gesellschaftliche Intoleranz oder Gewalt gegenüber religiösen Minderheiten. Es sollte untersucht werden, inwieweit sich dies auch auf die Arbeit der Alumni vor Ort auswirkt, zumal die staatliche und gesellschaftliche Intoleranz gegenüber religiösen Minderheiten weltweit zunimmt. Alle fünf Länder wurden im Rahmen von drei- bis achtwöchigen Feldaufenthalten besucht, um dort die Alumni und Partner*innen des KAAD innerhalb der Strukturen vor Ort zu erleben und zu interviewen. Zusätzlich wurden Interviews mit Studierenden und Almuni sowie mit den Programmverantwortlichen in Deutschland durchgeführt.

Der erste Feldaufenthalt fand in Georgien im September und Oktober $2016 \mathrm{im}$ Rahmen eines Forschungsaufenthaltes an der KAAD-Partneruniversität SukhanSaba Orbeliani Universität in Tiflis statt. Die Interviews mit Alumni des KAAD wurden vor allem in Tiflis und vereinzelt in anderen Städten und Regionen des Landes (wie in Kachetien) geführt. Auch Vertreter*innen verschiedener Universitäten (wie der Sulkhan-Saba Orbeliani Universität oder der Staatlichen Universität Tiflis), staatlicher Einrichtungen und deutscher entwicklungsrelevanter Organisationen und Institutionen - u. a. DAAD, Goethe-Institut und Konrad-Adenauer-Stiftung - sowie ehemalige georgische Studierende, die in Deutschland studiert haben, dabei aber nicht vom KAAD gefördert wurden, wurden interviewt. Zudem wurden teilnehmende Beobachtungen in Universitäten, staatlichen Einrichtungen und anderen relevanten Organisationen und Institutionen durchgeführt sowie an verschiedenen Veranstaltungen teilgenommen, wie z. B. einem KAAD-Alumnitreffen 
in der Orbeliani Universität in Tiflis. Auch der damals stattfindende Besuch des Papstes im Micheil-Meschi-Stadion wurde von uns beobachtet.

Der zweite Feldaufenthalt wurde in Israel und Palästina im November und Dezember 2016 unternommen. Die Interviews und Beobachtungen wurden in verschiedenen Städten Israels und Palästinas durchgeführt. Die ersten Interviews wurden in Tel Aviv gemacht mit Vertreter*innen deutscher Institutionen und Organisationen, die in der Entwicklungs- und Forschungszusammenarbeit tätig sind (u. a. DAAD, FES und HBS) sowie israelischer Institutionen (u. a. der Universität Tel Aviv). Die meisten Interviews mit KAAD-Alumni wurden in der Region um Bethlehem (Bethlehem, Beit Jala und Beit Sahour) durchgeführt, weil sich dort mit der Bethlehem Universität die Partneruniversität des KAAD befindet und dort die meisten Alumni des KAAD leben und arbeiten. Weitere Interviews mit Alumni wurden in der palästinensischen Hauptstadt Ramallah durchgeführt. Zudem wurden in Bethlehem und Umgebung ehemalige palästinensische Studierende, die nicht vom KAAD gefördert wurden, sowie Vertreterinnen von palästinensischen Organisationen und Institutionen, Krankenhäusern, Kirchen und NGO's interviewt. Teilnehmende Beobachtungen wurden in verschiedenen privaten und staatlichen Krankenhäusern durchgeführt, um die Ergebnisse der Interviews besser einordnen zu können und um einen Vergleich der Krankenversorgung und -behandlung zu haben. Ferner wurden auch Beobachtungen an der KAAD-Partneruniversität in Bethlehem sowie in Kirchen und staatlichen Einrichtungen gemacht (wie z. B. im palästinensischen Gesundheitsministerium in Ramallah).

Die sechswöchige Feldforschung in Indonesien im Mai und Juni 2017 wurde im Rahmen eines Forschungsaufenthalts an der KAAD-Partneruniversität Atma Jaya Universität Jakarta unternommen. Mehr als zwei Drittel unserer Interviews mit indonesischen KAAD-Alumni wurden in Jakarta durchgeführt, wo sich die meisten Alumni nach ihrer Rückkehr niedergelassen haben. Ferner wurden auch Interviews mit Alumni in der zweitgrößten Stadt Indonesiens, Jogyakarta, sowie während einer KAAD-Alumi-Tagung in Pontianak auf der Insel Borneo im Juni 2017 durchgeführt. Wie auch in den anderen Fallstudienländern wurden Interviews mit ehemaligen indonesischen Studierenden in Deutschland durchgeführt, die ein Stipendium einer anderen Stiftung erhalten oder ohne Stipendium in Deutschland studiert haben. Ferner wurden auch Interviews mit Vertreter*innen deutscher Organisationen und Institutionen in Indonesien gemacht, darunter GIZ, DAAD, KAS und FES. Diese Interviews dienten einem besseren Verständnis der sozialen und wirtschaftlichen Lage in Indonesien und der besonderen Situation der Rückkehrer*innen. Um die Ergebnisse besser einordnen zu können, wurden auch hier Beobachtungen in Unternehmen der Alumni sowie in Einrichtungen deutscher Organisationen und Institutionen in Indonesien durchgeführt. 
Die vierte Forschungsreise wurde in Kolumbien von Oktober bis Dezember 2017 im Rahmen eines Forschungsaufenthaltes an der Universidad Javeriana in Bogotá unternommen. Die meisten Alumni wurden in der Landeshauptstadt Bogotá, die das politische und wirtschaftliche Zentrum des Landes darstellt, befragt. Zudem auch Alumni in Medellin, der zweitgrößten Stadt des Landes. Interviews mit ehemaligen Studierenden, die nicht vom KAAD gefördert wurden, wurden ebenfalls durchgeführt. Ähnlich wie in den anderen Fallstudienländern, wurden auch Interviews mit Vertreter*innen des DAAD, Goethe-Instituts sowie der Friedrich-Ebert-Stiftung und Heinrich-Böll-Stiftung durchgeführt. Zudem wurden Beobachtungen an Universitäten, Unternehmen und staatlichen Einrichtungen in Bogotá und Medellin durchgeführt.

Die fünfte und letzte Feldforschung wurde in Ghana im Rahmen eines Forschungsaufenthalts an der Kwame-Nkruma-Universität in Kumasi (KNUST) im Februar und März 2018 durchgeführt. Im Rahmen unserer Studie wurden Alumni zunächst während eines Aufenthalts in der Hauptstadt Accra interviewt, danach in Kumasi, der zweitgrößten Stadt des Landes, und anschließend in Tamale, einer Universitätsstadt im Norden des Landes, wo viele KAAD-Alumni als Forschende und Mitarbeitende an der Universität tätig sind. In Accra und Tamale wurden zudem ehemalige Studierende ohne KAAD-Stipendium interviewt. Ferner wurden auch ergänzende Interviews und Gespräche mit Vertreter*innen des DAAD, GoetheInstituts, der GIZ sowie anderer relevanter Organisationen und Institutionen, wie der KAS geführt, die zu einem besseren Verständnis der entwicklungspolitischen Situation in Ghana beitragen sollten. ${ }^{7}$

Zusätzlich wurden Interviews in Deutschland durchgeführt. Um die Strukturen und Arbeit des KAAD zu verstehen, wurden alle Mitarbeitende der KAAD-Geschäftsstelle in Bonn interviewt sowie mehrere Gremienmitglieder des KAAD. Diese Interviews wurden direkt in der Geschäftsstelle des KAAD oder im Rahmen der KAAD-Jahresakademien in Bonn in den Jahren 2016, 2017 und 2018 (jeweils Ende April oder Anfang Mai) geführt. Ferner wurden auch ergänzende Interviews mit Vertreter*innen der katholischen Hochschulgemeinden in Bonn und Münster durchgeführt, um das Auswahlverfahren des KAAD zu besser verstehen. Wie in den Herkunftsländern, wurden auch Alumni aus allen fünf Fallstudienländern, die nach dem Studium nicht in ihr Herkunftsland zurückgekehrt, sondern in Deutschland geblieben sind, interviewt. Zudem wurden auch aktuelle Stipendiat*innen des KAAD befragt. Diese Interviews wurden entweder direkt im Studienort oder per Skype durchgeführt.

7 Detaillierte Auflistungen aller durchgeführten Interviews und Beobachtungen sind im Anhang. 
In diesem Rahmen wurden insgesamt 245 Interviews realisiert, davon mit 87 KAAD-Alumni in den fünf Fallstudienländern, 48 mit aktuellen Stipendiat*innen bzw. Alumni in Deutschland, 18 mit Mitarbeitenden des KAAD in Bonn und mit weiteren Partner*innen des KAAD in Deutschland sowie weitere 50 mit Vertreter*innen von KAAD-Partnerorganisationen und anderen Institutionen der Entwicklungszusammenarbeit in den Fallstudienländern. Dabei wurden 42 Interviews mit (ehemaligen) Studierenden aus den fünf Fallstudienländern, die nicht vom KAAD gefördert wurden, durchgeführt (Vergleichsgruppe). Die Interviews wurden auf Deutsch, Englisch und Spanisch durchgeführt. Ergänzt wurde die Feldforschung durch die erwähnten Beobachtungen, wie z. B. im Rahmen von Veranstaltungen der Alumni, an Universitäten, staatlichen Institutionen, Krankenhäusern, Kirchen, Kanzleien, Unternehmen usw. (insgesamt 58 Beobachtungen).

Tab. 1 Anzahl durchgeführter Interviews nach Fallstudienland und Interviewgruppe

\begin{tabular}{|c|c|c|}
\hline Land & Interviewgruppe & Anz. d. Interviews \\
\hline Israel/ & Palästinensische KAAD-Studierende/Alumni & 26 \\
\hline \multirow[t]{2}{*}{ Palästina } & $\begin{array}{l}\text { Ehemalige palästinensische Studierende ohne Stipen- } \\
\text { dium (Vergleichsgruppe) }\end{array}$ & 11 \\
\hline & $\begin{array}{l}\text { Entwicklungsrelevante Organisationen und Institutio- } \\
\text { nen in Israel/Palästina }\end{array}$ & 13 \\
\hline \multirow[t]{3}{*}{ Indonesien } & Indonesische KAAD-Studierende/Alumni & 32 \\
\hline & $\begin{array}{l}\text { Ehemalige indonesische Studierende ohne Stipendium } \\
\text { (Vergleichsgruppe) }\end{array}$ & 8 \\
\hline & $\begin{array}{l}\text { Entwicklungsrelevante Organisationen und Institutio- } \\
\text { nen in Indonesien }\end{array}$ & 6 \\
\hline \multirow[t]{3}{*}{ Ghana } & Ghanaische KAAD-Studierende/Alumni & 30 \\
\hline & $\begin{array}{l}\text { Ehemalige ghanaische Studierende ohne Stipendium } \\
\text { (Vergleichsgruppe) }\end{array}$ & 4 \\
\hline & $\begin{array}{l}\text { Entwicklungsrelevante Organisationen und Institutio- } \\
\text { nen in Ghana }\end{array}$ & 10 \\
\hline \multirow[t]{3}{*}{ Georgien } & Georgische KAAD-Studierende/Alumni & 15 \\
\hline & $\begin{array}{l}\text { Ehemalige georgische Studierende ohne Stipendium } \\
\text { (Vergleichsgruppe) }\end{array}$ & 14 \\
\hline & $\begin{array}{l}\text { Entwicklungsrelevante Organisationen und Institutio- } \\
\text { nen in Georgien }\end{array}$ & 8 \\
\hline \multirow[t]{5}{*}{ Kolumbien } & Kolumbianische KAAD-Studierende/Alumni & 32 \\
\hline & $\begin{array}{l}\text { Ehemalige kolumbianische Studierende ohne Stipen- } \\
\text { dium (Vergleichsgruppe) }\end{array}$ & 5 \\
\hline & $\begin{array}{l}\text { Entwicklungsrelevante Organisationen und Institutio- } \\
\text { nen in Kolumbien }\end{array}$ & 13 \\
\hline & Mitarbeitende und Mitglieder des KAAD & 18 \\
\hline & Insgesamt & 245 \\
\hline
\end{tabular}

Quelle: Eigene Zusammenstellung 


\subsection{Dokumentenanalyse und quantitative Befragung}

Parallel zu den Länderfallstudien wurde in der ersten Projektphase ein umfassender Überblick über die Geschichte des KAAD im Allgemeinen und über die Förderungsprogramme und konkrete Alumni-Arbeit des KAAD im Besonderen gewonnen. Hierzu wurden die organisationsinternen Daten, Statistiken und $\mathrm{Pu}-$ blikationen des KAAD analysiert, v. a. Stipendien-Datenbanken ${ }^{8}$, Jahresberichte, Tagungsberichte, Arbeitspapiere, Tagungsberichte, die sich im KAAD-Archiv in Bonn befinden sowie Unterlagen und Veröffentlichungen der Alumni-Vereine in den Fallstudienländern. Des Weiteren wurden intensive Länderrecherchen in Bezug auf Politik, Wirtschaft, Gesundheitswesen, Bildungs- und Wissenschaftssystem sowie die Migrationspolitik des jeweiligen Fallstudienlandes durchgeführt. Neben der Literatur- und Dokumentenanalyse wurden auch explorative Experteninterviews durchgeführt, insbesondere mit KAAD-Verantwortlichen und Vertreter*innen von Alumni-Vereinen. Die Interviews dienten der Erschließung des Themas sowie einem besseren Verständnis der Arbeit des KAAD und zur Vorbereitung vertiefender Interviews mit den Alumni.

Ergänzt wurde die Untersuchung durch eine quantitative Befragung aller (erreichbarer) Alumni und aktueller Stipendiat*innen des KAAD in Form einer Online-Befragung. Mit der quantitativen Online-Befragung wurde erst begonnen, als bereits ein Teil der Interviews (sowohl in Deutschland als auch in ein paar Herkunftsländern) gemacht wurden, damit Fragen in die Befragung integriert werden konnten, die vorher noch nicht auftauchten und sich erst im Laufe der Interviews als wichtig herausstellten. Bei der Untersuchung wurden insgesamt 3.347 Stipendiat*innen sowie Alumni angeschrieben, wovon 569 geantwortet haben, so dass die Rücklaufquote rund 17 Prozent betrug. Die Teilnehmenden der Online-Befragung waren zu 57 Prozent männlich und zu 43 Prozent weiblich und zwischen 21 und 79 Jahre alt. Der Altersdurchschnitt betrug 40 Jahre. Der Großteil der Befragten war katholisch (70\%). Die restlichen 30 Prozent waren überwiegend christlich-orthodox (ca. $14 \%)$, evangelisch (4\%) sowie sunnitisch (2\%) oder schiitisch (0,5\%). Und nur ein sehr kleiner Teil war entweder buddhistisch oder hinduistisch (unter 0,5\%). Nur knapp 4 Prozent aller Teilnehmenden gehören keiner Konfession an. Die Teilnehmenden kamen aus 61 Ländern, davon die meisten aus den Fallstudienländern Kolumbien (8\%), Ghana (7\%) und Indonesien (5\%). Ferner auch aus

8 Die Stipendien-Datenbanken lieferten uns wichtige Hintergrundinformationen zu den Alumni und Stipendiat*innen in Bezug auf Geschlecht, Konfession, Herkunft, Bewerbungsmotivation, Studien- und Forschungsschwerpunkte sowie auf ehrenamtliches Engagement und Netzwerkaktivitäten. 
Polen (knapp $5 \%$ ), der Ukraine (4\%), Kenia (4\%), Ecuador (3,5\%), Israel/Palästina (3,0\%), Guatemala (3,0\%) und Rumänien (3,0\%). Davon besitzen die allermeisten ausschließlich einen Pass ihres Herkunftslandes (94\%), und nur knapp zwei Prozent besitzen nur einen deutschen Pass. Knapp vier Prozent besitzen sowohl einen deutschen als auch einen ausländischen Pass. Die Studien- bzw. Forschungsaufenthalte der Stipendiat*innen in Deutschland wurden hauptsächlich in den letzten 20 Jahren durchgeführt.

Die Fragen des Online-Fragebogens bezogen sich in erster Linie auf das Leben und die Erwartungen vor dem Studium in Deutschland (Schule und Studium im Herkunftsland, Motivation für ein Studium in Deutschland, Erwartungen an das Leben und das Studium in Deutschland etc.), die Erfahrungen während des Studiums (Studienzeit, Stipendium, Tätigkeiten neben dem Studium, Erfahrungen in der deutschen Gesellschaft, Kontakte während des Studiums in das Herkunftsland, Herausforderungen etc.) und (ggf.) die weitere Entwicklung nach dem Studium, also nach der Rückkehr ins Herkunftsland (Arbeit, soziales Engagement, weitere Beziehungen nach Deutschland, Herausforderungen etc.), dem Verbleib in Deutschland oder der Weiterwanderung in ein anderes Land (Arbeit, Engagement, weitere Kontakte in das Herkunftsland, Herausforderungen etc.). Dieser Struktur folgt der nachfolgende Ergebnisteil unserer Untersuchung. Zudem lassen wir im Folgenden die Stipendiat*innen häufig selbst zu Wort kommen, um dem stark empirischen Charakter der Studie Rechnung zu tragen.

Open Access Dieses Kapitel wird unter der Creative Commons Namensnennung 4.0 International Lizenz (http://creativecommons.org/licenses/by/4.0/deed.de) veröffentlicht, welche die Nutzung, Vervielfältigung, Bearbeitung, Verbreitung und Wiedergabe in jeglichem Medium und Format erlaubt, sofern Sie den/die ursprünglichen Autor(en) und die Quelle ordnungsgemäß nennen, einen Link zur Creative Commons Lizenz beifügen und angeben, ob Änderungen vorgenommen wurden.

Die in diesem Kapitel enthaltenen Bilder und sonstiges Drittmaterial unterliegen ebenfalls der genannten Creative Commons Lizenz, sofern sich aus der Abbildungslegende nichts anderes ergibt. Sofern das betreffende Material nicht unter der genannten Creative Commons Lizenz steht und die betreffende Handlung nicht nach gesetzlichen Vorschriften erlaubt ist, ist für die oben aufgeführten Weiterverwendungen des Materials die Einwilligung des jeweiligen Rechteinhabers einzuholen. 